\title{
The value of enhanced computed tomography combined with magnetic resonance imaging in the differential diagnosis of thymomas and thymic cysts before operation
}

\author{
Xuesong Zhang ${ }^{1 \wedge}$, Ruiyu Zhang ${ }^{1}$, Yang $\mathrm{Cao}^{2}$, Xiaomeng Wang ${ }^{2}$, Yuan Chen ${ }^{1}$ \\ ${ }^{1}$ Department of Cardiothoracic Surgery, General Hospital of Tianjin Medical University, Tianjin, China; ${ }^{2}$ Department of Imaging, General Hospital \\ of Tianjin Medical University, Tianjin, China \\ Contributions: (I) Conception and design: Y Chen, X Zhang; (II) Administrative support: Y Chen; (III) Provision of study materials or patients: Y Cao, \\ X Wang; (IV) Collection and assembly of data: X Zhang, R Zhang; (V) Data analysis and interpretation: X Zhang, R Zhang; (VI) Manuscript writing: \\ All authors; (VII) Final approval of manuscript: All authors. \\ Correspondence to: Yuan Chen. Department of Cardiothoracic Surgery, General Hospital of Tianjin Medical University, No. 154 Anshan Road, \\ Heping District, Tianijn, 300052, China. Email: yuanchen@tmu.edu.cn.
}

Background: We evaluated the application of enhanced computed tomography (CT) combined with magnetic resonance imaging (MRI) to preoperative diagnosis of thymomas and thymic cysts.

Methods: The patients admitted to General Hospital of Tianjin Medical University with anterior mediastinal masses (maximum diameter $<5 \mathrm{~cm}$ ) from 2016 to 2020 were analyzed. In total, 81 patients had been included, including 43 (13 male, 30 female) thymomas (28 cases of myasthenia gravis) and 38 (17 male, 21 female) thymic cysts ( 2 cases of myasthenia gravis). The median age of the patients with thymomas and thymic cysts was 56 (48-65) and 56.5 (50.8-64) years, respectively. The features of thymomas and thymic cysts revealed on enhanced CT, MRI, and clinically were compared, and we evaluated the diagnostic performance of enhanced CT combined with MRI.

Results: Most thymomas exhibited iso-signal intensity on T1- and T2-weighted imaging (T2WI) and isoor high-signal intensity on diffusion-weighted imaging. In masses with diameters $<3 \mathrm{~cm}$, diffusion-weighted imaging (DWI) and CT were not statistically significant $(\mathrm{P}>0.05)$. In masses with diameters of $<5$ and $<3 \mathrm{~cm}$, the area under the receiver operating characteristic curves (AUC) of the enhanced 120sCT value were 0.94 and 0.95 , respectively, and those for apparent diffusion coefficient (ADC) value were 0.92 and 0.91 , respectively. In masses with diameters $<5 \mathrm{~cm}$, the diagnostic rates of enhanced CT, MRI, and enhanced CT combined with MRI for thymomas were 40.5\%, 64.9\%, and 70.3\%, respectively, and those for thymic cysts were $37.5 \%, 62.5 \%$, and $71.9 \%$, respectively. In masses with diameters $<3 \mathrm{~cm}$, the diagnostic rates of enhanced CT, MRI, and enhanced CT combined with MRI for thymomas were 40.9\%, 68.2\%, and 77.3\%, respectively, and those for thymic cysts were $32.0 \%, 64.0 \%$, and $68.0 \%$, respectively.

Conclusions: Enhanced CT combined with MRI is superior to enhanced CT or MRI alone for preoperative differential diagnosis of thymomas and thymic cysts with diameters of $<5$ and $<3 \mathrm{~cm}$.

Keywords: Differential diagnosis; enhanced CT; magnetic resonance imaging (MRI); thymic cyst; thymoma

Submitted Jan 16, 2021. Accepted for publication Apr 27, 2021.

doi: $10.21037 /$ tcr-21-96

View this article at: https://dx.doi.org/10.21037/tcr-21-96

^ ORCID: 0000-0002-9064-1359. 


\section{Introduction}

The use of CT in health examinations has been increasing. Therefore, clinical discovery of mediastinal lesions has gradually increased over the past several years. Thymomas account for $20 \%$ of all mediastinal tumors (1), and thymic cysts account for $1-3 \%$ (2). Considering the limitations of pathological examinations such as tumor biopsy, imaging examinations (especially CT examinations) have become the most important basis for preoperative diagnosis (3). In a study on 56,358 patients who underwent CT scans, Yoon et al. reported that $32(64 \%)$ patients were diagnosed with thymic cysts out of 50 who underwent anterior mediastinal mass resection (4), resulting in $22-44 \%$ of patients undergoing non-therapeutic thymectomy (5). Therefore, it is difficult to distinguish between thymomas and thymic cysts based on CT features alone, especially thymomas and thymic cysts with diameters of $<28 \mathrm{~mm}$ (6-8), which can cause misdiagnosis. The main reason for such misdiagnosis is that the cyst contains high levels of protein, which may cause secondary bleeding, and the higher protein level causes the density of thymic cysts to be higher than that of simple cysts (9).

Thanks to continuous advancement of its technology, MRI has been increasingly used for diagnosis of anterior mediastinal diseases. Traditional MRI can provide information about the location, outline, and boundary of mediastinal masses, and it has better soft tissue contrast. The application of MRI on other parts of the body has also been very successful. The MRI technique called DWI can also display cell density and water fluidity. Its advantage compared with CT is that it may provide useful information about the status of patients for whom the use of intravenous contrast agents is contraindicated $(10,11)$. Great progress has been made in MRI, and the use of stronger field gradients, parallel imaging technology, multichannel coils, and planar echo MRI has been extended to mediastinal diseases $(9,11,12)$. Compared with CT, MRI is better at detecting cystic lesions such as thymic cysts and distinguishing cysts from solid masses (13).

However, few practitioners use enhanced CT combined with MRI for differential diagnosis of thymomas and thymic cysts with diameters of $<5 \mathrm{~cm}$. The purpose of this study is to evaluate the accuracy of enhanced CT combined with MRI in the diagnosis of thymomas and thymic cysts.

We present the following article in accordance with the STARD reporting checklist (available at https://dx.doi. org/10.21037/tcr-21-96).

\section{Methods}

\section{General information on included patients}

Inclusion criteria: (I) age 18-75 years, with no gender limitation; (II) thymomas or thymic cysts with confirmed pathology; (III) patients who underwent both enhanced CT and MRI; (IV) average interval between pathological diagnosis and imaging examination of no more than 1.5 months.

Exclusion criteria: (I) thymomas or thymic cysts of diameter $>5 \mathrm{~cm}$; (II) imaging showed that the mass had invaded the pericardium, lung, large blood vessels, etc.; (III) thymomas or thymic cysts with retrosternal goiter, lymphoma, mediastinal germ cell tumor, etc.

We performed a retrospective analysis of 279 patients with tumors of the anterior mediastinum who were admitted to the General Hospital of Tianjin Medical University from May 1, 2016 to April 30, 2020. All patients received surgical treatment and pathological examination. After screening, 81 cases ( 43 cases of thymoma and 38 cases of thymic cyst) met the research criteria. However, not all patients have done every check, and some patients took CT scans in other institutions. So patients with a lack of data are excluded from the study. Two thoracic radiologists, one senior and the other junior, made a coincident diagnosis of effects of the CT and MRI without knowledge of clinical and pathologic data of those subjects. The senior radiologist measured the CT value by placing a round region of interest (ROI) covering the largest area of thymic lesions. If the difference between enhanced CT and plain scan CT value is greater than 15 , we consider the lesion to be enhanced.

\section{Imaging manifestations (14)}

Thymoma in CT: It appears in one or two thymus lobes in a multi-lobed form with fine linear calcification or necrosis inside or on the periphery, uneven density, and unclear boundaries, changing the outline of the normal thymus and diffusing to the pleura, pericardium and mediastinum.

Thymic cyst in CT: It is usually unilateral, small with thin wall, uniform density, and occasionally thin soft tissue septum. It mostly possess a mixed density when calcified or bleeding inside.

Thymoma in MRI: The signal on T1WI and T2WI is comparable between muscle and fat, accompanied by mild heterogeneity and often with fibrous spacing. The signal is uneven due to necrosis and hemorrhage and even more uneven if it is aggressive. On T2WI, there are 
compartments and lobulate structures with uneven signal intensity in the leaf.

Thymic cyst in MRI: The signal is lower than that of muscle on T1WI, and higher than that of fat on T2WI. If there is inflammation, infection, or bleeding, it manifested iso- or low signal mixed with high signal on T2WI.

We compared both the clinical and imaging characteristics of patients with thymomas and thymic cysts through enhanced CT combined with MRI. The method of calculating diagnostic rate for CT and MRI is as follows: The diagnostic rate of CT is presented as the ratio of the number of patients with thymoma or thymic cyst diagnosed correctly by only one imaging method of CT to the number of patients with thymoma or thymic cyst confirmed by pathology in this study. MRI is consistent with the above. The diagnostic rate of CT combined with MRI is presented as the ratio of the number of patients with thymoma or thymic cyst diagnosed correctly by either CT or MRI to the number of patients with thymoma or thymic cyst confirmed by pathology.

\section{Enhanced CT scan}

A CT system (GE Discovery CT750 HD, GE HealthCare, USA) was used for imaging, with supine position inspiration and breath-hold scanning, the scan range spanned from the lung tip to the lung base (scanning layer thickness: $1.25 \mathrm{~mm}$; tube voltage: $120 \mathrm{kV}$; tube current: $100-250 \mathrm{~mA}$ (automatic dose adjustment); and matrix: $512 \times 512$ ). Then, iohexol $(70 \mathrm{~mL}$, rate of $3.5-4.0 \mathrm{~mL} / \mathrm{s})$ was given to obtain enhanced CT. Enhanced CT scan delay time is 30 s and 120 s after the injection of the iohexol.

\section{MRI}

A 3.0T MRI device (Siemens Magnetom Prisma, Germany) was used for imaging, with supine position inspiration and breath-hold scanning, the scan range spanned from the lung tip to the lung base. T1WI: BH Ax 2D T1 in phase; TR, 270 ms; TE, 2.24 ms; matrix, 256×192. T2WI: RTr Ax T2FSE ASSET; TR, 3,000-5,000 ms; TE, $85 \mathrm{~ms}$; matrix, $288 \times 256$. DWI: matrix, $128 \times 128$; b-value, $800 \mathrm{~s} / \mathrm{mm}^{2}$. The thickness of the three series is $6 \mathrm{~mm}$.

\section{Research indicators}

Main indicator: Diagnosis rate of enhanced CT combined with MRI (either enhanced CT or MRI is diagnosed correctly, or both methods are diagnosed correctly).

Secondary indicators:

(I) Patient characteristics: gender, age, whether combined with myasthenia gravis;

(II) Tumor morphological characteristics: tumor location, outline, boundary, shape;

(III) Enhanced CT performance: whether CT is enhanced, whether the enhancement is uniform, and the CT value;

(IV) AUC of plain CT value, enhanced CT value, and $\triangle \mathrm{CT}$ value;

(V) MRI manifestations: T1WI, T2WI, DWI.

\section{Statistical analysis}

All data were analyzed using SPSS version 23.0 (SPSS, Inc., Chicago, IL, USA). Age was represented by the median (interquartile range), and the Mann-Whitney test was used for its analysis. Gender, whether or not masses were combined with myasthenia gravis, location, outline, boundary, shape, and qualitative features of CT and MRI were analyzed by chi-square or Fisher's exact tests. The CT values of plain CT, enhanced 30sCT, enhanced 120sCT, enhanced 30s $\Delta \mathrm{CT}$, and enhanced $120 \mathrm{~s} \Delta \mathrm{CT}$ scans and $\mathrm{ADC}$ values were analyzed by Student's $t$-tests. The AUC curve was used to evaluate the feasibility of using those CT values and $\mathrm{ADC}$ values for the differential diagnosis of thymomas and thymic cysts. $\mathrm{P}<0.05$ was considered to indicate a statistically significant difference.

\section{Ethical statement}

The study was conducted in accordance with the Declaration of Helsinki (as revised in 2013). The study does not require ethical approval. Because it is a retrospective study, it only needs to review the patient's preoperative imaging data and postoperative pathological results, and does not need to use the patient's blood and tissue specimens, so it does not involve ethical issues and individual consent for this retrospective analysis was waived.

\section{Results}

\section{General characteristics of included patients}

In total, 81 patients had masses with diameters of $<5 \mathrm{~cm}$, including 43 (13 male, 30 female) thymomas (28 cases of myasthenia gravis) and 38 (17 male, 21 female) thymic cysts 
Table 1 General characteristics of the patients

\begin{tabular}{|c|c|c|c|c|c|c|}
\hline Characteristics & \multicolumn{2}{|c|}{ Thymic cyst } & \multicolumn{2}{|c|}{ Thymoma } & $\mathrm{P}^{\dagger}$ & $\mathrm{P}^{\ddagger}$ \\
\hline Gender, n (\%) & & & & & 0.180 & 0.404 \\
\hline Male & $17(44.7)$ & $10(33.3)$ & $13(30.2)$ & $5(22.7)$ & & \\
\hline Female & $21(55.3)$ & $20(66.7)$ & $30(69.8)$ & $17(77.3)$ & & \\
\hline Myasthenia gravis, n (\%) & & & & & $<0.001$ & $<0.001$ \\
\hline Yes & $2(5.2)$ & $1(3.3)$ & $28(65.1)$ & $15(68.1)$ & & \\
\hline No & $36(94.8)$ & $29(96.7)$ & $15(34.9)$ & 7 (31.9) & & \\
\hline
\end{tabular}

${ }^{\dagger},<5 \mathrm{~cm} ;{ }^{\ddagger},<3 \mathrm{~cm}$.

( 2 cases of myasthenia gravis). According to the World Health Organization (WHO) classification, thymomas were classified as $\mathrm{A}$ in 4 (9.3\%) patients, $\mathrm{AB}$ in 15 (34.9\%), B1 in 7 (16.3\%), B2 in 13 (30.2\%), and B3 in 4 (9.3\%). The median age of the patients with thymomas and thymic cysts was 56 (48-65) and 56.5 (50.8-64) years, respectively. There were no statistically significant differences between the gender or age of patients who had thymomas and thymic cysts $(\mathrm{P}>0.05)$. Among the patients with thymic cysts, 94.8\% did not have myasthenia gravis, whereas the proportion was $34.9 \%$ in patients with thymomas, and this difference was statistically significant $(\mathrm{P}<0.05$; Table 1).

In total, 52 patients had masses with diameters of $<3 \mathrm{~cm}$, including 22 (5 male, 17 female) thymomas (15 cases of myasthenia gravis) and 30 (10 male, 20 female) thymic cysts ( 1 case of myasthenia gravis). The median age of the patients with thymomas and thymic cysts was 57.5 (50.5-65) and 55 (46.8-64) years, respectively. There were no statistically significant differences between the gender and age of the patients who had thymomas and thymic cysts ( $\mathrm{P}>0.05$ ). Among the patients with thymic cysts, $96.7 \% \mathrm{did}$ not have myasthenia gravis, whereas the proportion was $31.9 \%$ in patients with thymomas, and this difference was statistically significant $(\mathrm{P}<0.05$; Table 1$)$.

\section{Imaging features}

Among thymic tumors with diameters of $<5 \mathrm{~cm}$, the outline of the tumor is a meaningful identification index. The proportions of smooth outline in thymomas and thymic cysts were $44.1 \%$ and $84.2 \%$, respectively, and the difference between the two lesion types was statistically significant $(\mathrm{P}<0.05$; Table 2). Thymomas were enhanced on enhanced CT, had iso-signals on T1 and T2WI, and had iso- or high-signal intensity on DWI to a greater degree than thymic cysts. The differences in these indicators between the two mass types were statistically significant $(\mathrm{P}<0.05$; Table 3). However, the location, boundary, and shape of the masses showed no statistically significant differences between thymomas and thymic cysts $(\mathrm{P}>0.05)$.

The tumor outline is also a meaningful identification index among thymic tumors with diameters of $<3 \mathrm{~cm}$. The proportion of smooth outline in thymomas and thymic cysts with diameters of $<3 \mathrm{~cm}$ was $36.3 \%$ and $83.3 \%$, respectively, and the difference between the two lesion types was statistically significant $(\mathrm{P}<0.05$; Table 4$)$. Thymomas were enhanced and unevenly enhanced on enhanced CT, had iso-signals on T1WI and T2WI, and had iso- or highsignal intensity on DWI to a greater degree than thymic cysts. However, only T1WI and T2WI reached statistical significance (Table 5). Moreover, the location, boundary, shape, and DWI intensity of the masses had no statistically significant differences between thymomas and thymic cysts $(\mathrm{P}>0.05)$.

We measured the ADC values of thymoma and thymic cyst. The ADC values of thymoma and thymic cyst less than $5 \mathrm{~cm}$ were $1.79 \times 10^{-3} \mathrm{~mm}^{2} / \mathrm{s} \pm 0.84 \times 10^{-3} \mathrm{~mm}^{2} / \mathrm{s}$ and $3.28 \times 10^{-3} \mathrm{~mm}^{2} / \mathrm{s} \pm 0.57 \times 10^{-3} \mathrm{~mm}^{2} / \mathrm{s}$, the ones that less than $3 \mathrm{~cm}$ are $1.91 \times 10^{-3} \mathrm{~mm}^{2} / \mathrm{s} \pm 0.84 \times 10^{-3} \mathrm{~mm}^{2} / \mathrm{s}$ and $3.29 \times 10^{-3} \mathrm{~mm}^{2} / \mathrm{s} \pm 0.64 \times 10^{-3} \mathrm{~mm}^{2} / \mathrm{s}$. The distinction of data, represented by Mean $\pm \mathrm{SD}$, is statistically significant. We made the receiver operating characteristic (ROC) curves of $\mathrm{ADC}$ value and discovered that when the lesion is less than $5 \mathrm{~cm}$ and $3 \mathrm{~cm}, \mathrm{ADC}>2.68 \times 10^{-3} \mathrm{~mm}^{2} / \mathrm{s}$ and $>2.55 \times 10^{-3} \mathrm{~mm}^{2} / \mathrm{s}$ are more prone to thymic cysts. And the AUCs with masses $<5$ and $<3 \mathrm{~cm}$ for ADC values were 0.92 and 0.91 , respectively 
Table 2 General imaging features of thymic cysts and thymomas with diameters of $<5 \mathrm{~cm}$

\begin{tabular}{|c|c|c|c|c|}
\hline Features & Thymic cyst $(n=38)$ & Thymoma $(n=43)$ & $\chi^{2}$ & $\mathrm{P}$ \\
\hline Left & $15(39.4)$ & $20(46.5)$ & & \\
\hline Middle & $11(28.9)$ & $10(23.2)$ & & \\
\hline Right & $12(31.7)$ & $13(30.3)$ & & \\
\hline Smooth & $32(84.2)$ & $19(44.1)$ & & \\
\hline Unsmooth & $6(15.8)$ & $24(55.9)$ & & \\
\hline Bound, n (\%) & & & 3.28 & 0.114 \\
\hline Clear & 37 (97.3) & $37(86.0)$ & & \\
\hline Lobulated/Irregular & $23(60.5)$ & $31(72.0)$ & & \\
\hline Round & $1(2.6)$ & $1(2.3)$ & & \\
\hline Oval & $14(36.9)$ & $11(25.7)$ & & \\
\hline
\end{tabular}

Table $3 \mathrm{CT}$ and MRI features of thymic cysts and thymomas with diameters of $<5 \mathrm{~cm}$

\begin{tabular}{|c|c|c|c|c|}
\hline Manifestation & Thymic cyst & Thymoma & $\chi^{2}$ & $P$ \\
\hline T1WI & & & 13.28 & 0.001 \\
\hline Middle (Longish, Shortish) & $11(37.9)$ & $30(81.1)$ & & \\
\hline Long & $13(44.8)$ & $6(16.2)$ & & \\
\hline T2WI & & & 9.28 & 0.010 \\
\hline Middle (Longish, Shortish) & $9(31.0)$ & $25(67.6)$ & & \\
\hline Long & $19(65.5)$ & $12(32.4)$ & & \\
\hline Short & $1(3.5)$ & 0 & & \\
\hline High & $5(17.2)$ & $20(54.1)$ & & \\
\hline Low & $13(44.9)$ & $2(5.4)$ & & \\
\hline CT, n (\%) & $(n=17)$ & $(n=23)$ & 7.73 & 0.009 \\
\hline Unenhanced & $5(29.4)$ & 0 & & \\
\hline Enhanced & $12(60.6)$ & $23(100.0)$ & 4.11 & 0.059 \\
\hline Balance & $6(50.0)$ & $4(17.4)$ & & \\
\hline Unbalance & $6(50.0)$ & $19(82.6)$ & & \\
\hline
\end{tabular}

\footnotetext{
${ }^{\dagger}$, signal intensity. CT, computed tomography; MRI, magnetic resonance imaging; DWI, diffusion-weighted imaging.
} 
Table 4 General imaging features of thymic cysts and thymomas with diameters of $<3 \mathrm{~cm}$

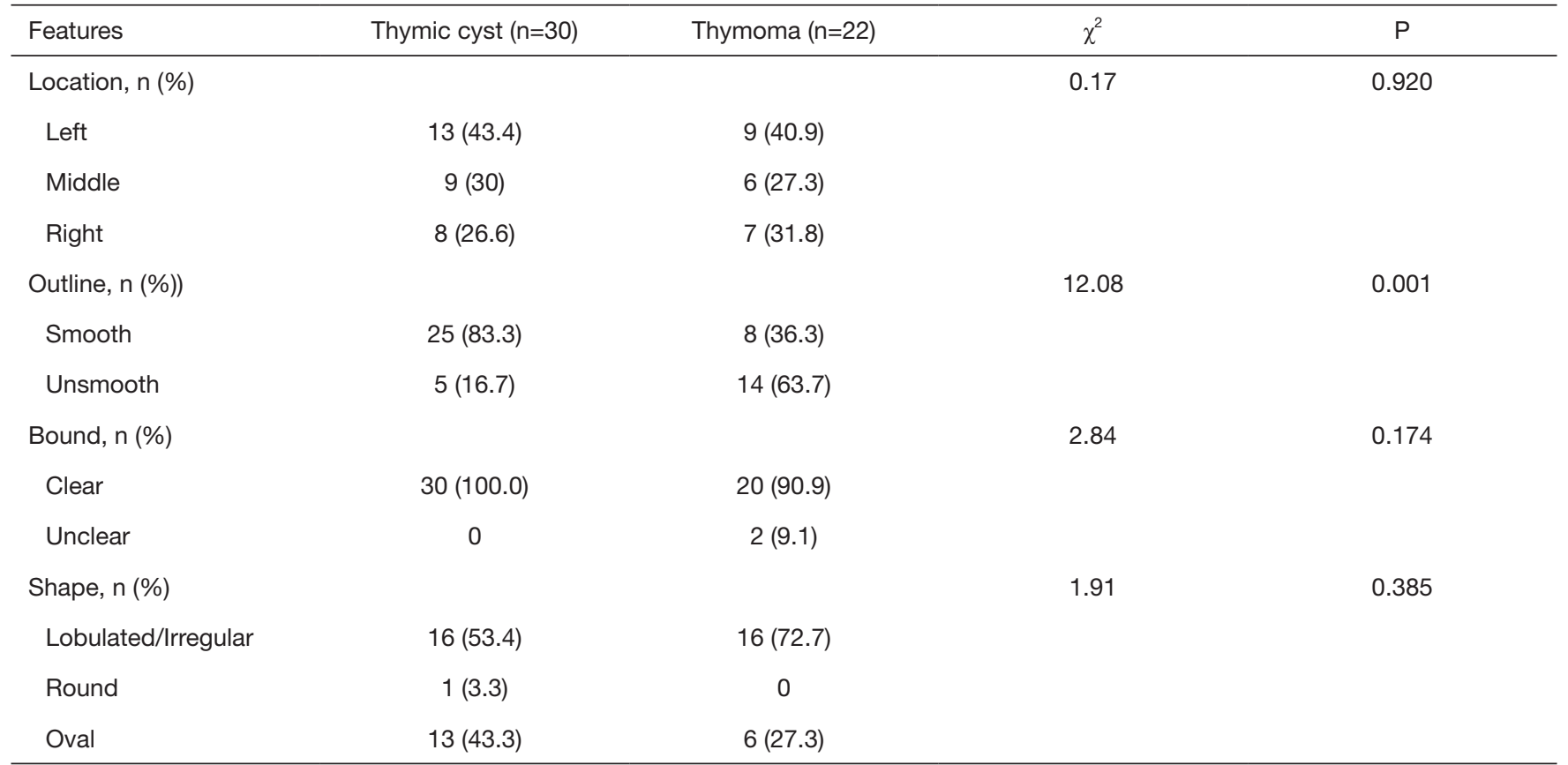

Table 5 CT and MRI features of thymic cysts and thymomas with diameters of $<3 \mathrm{~cm}$

\begin{tabular}{|c|c|c|c|c|}
\hline Manifestation & Thymic cyst & Thymoma & $\chi^{2}$ & $P$ \\
\hline T1WI & & & 9.92 & 0.007 \\
\hline Middle (Longish, Shortish) & $10(47.6)$ & $20(90.1)$ & & \\
\hline Long & $8(38.1)$ & $2(9.9)$ & & \\
\hline $\mathrm{T} 2 \mathrm{WI}$ & & & 8.95 & 0.003 \\
\hline Middle (Longish, Shortish) & $9(42.9)$ & $19(86.4)$ & & \\
\hline Long & $12(57.1)$ & $3(13.6)$ & & \\
\hline Short & 0 & 0 & & \\
\hline High & $5(23.8)$ & $9(40.9)$ & & \\
\hline Low & 7 (33.3) & $2(9.1)$ & & \\
\hline CT, n (\%) & $(n=15)$ & $(n=12)$ & 3.76 & 0.106 \\
\hline Unenhanced & $4(26.7)$ & 0 & & \\
\hline Enhanced & $11(73.3)$ & $12(100.0)$ & 2.10 & 0.214 \\
\hline Balance & $6(54.5)$ & $3(25.0)$ & & \\
\hline Unbalance & $5(45.5)$ & $9(75.5)$ & & \\
\hline
\end{tabular}

\footnotetext{
${ }^{\dagger}$, signal intensity. CT, computed tomography; MRI, magnetic resonance imaging; DWI, diffusion-weighted imaging.
} 
A

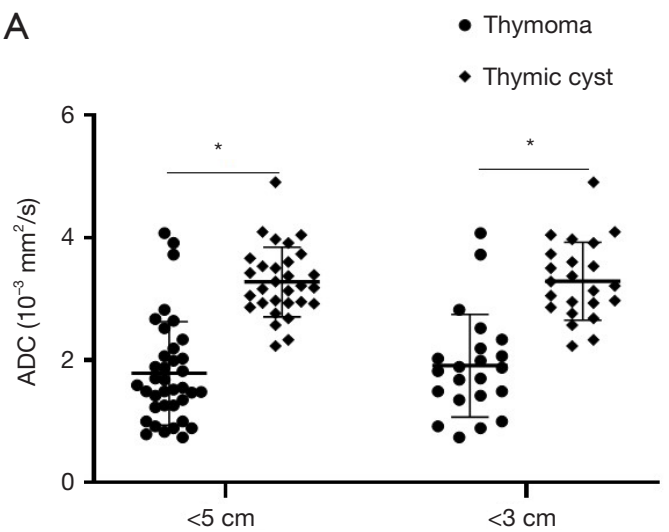

B

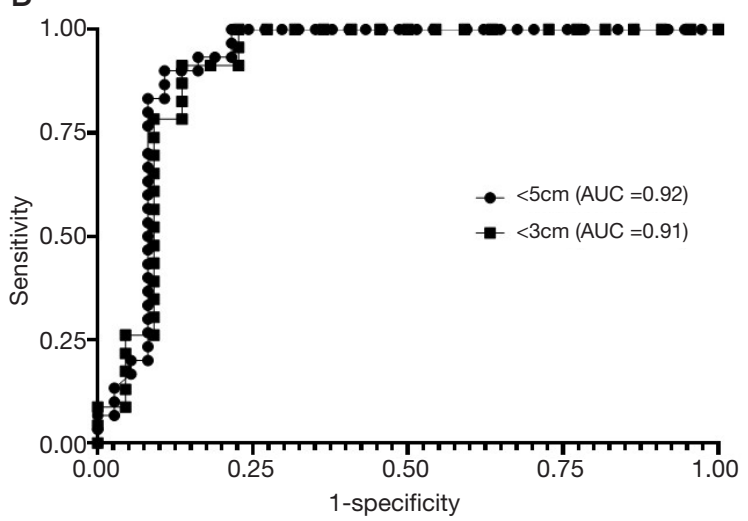

Figure 1 Scatter plots and ROC curves of the value of ADC of thymomas and thymic cysts. (A) Scatter plots of the value of ADC of thymomas and thymic cysts with diameters $<5$ and $<3 \mathrm{~cm}$. (B) ROC curves of the value of ADC of thymomas and thymic cysts with diameters $<5 \mathrm{~cm}$ and $<3 \mathrm{~cm}$. *, $\mathrm{P}<0.001$. ADC, apparent diffusion coefficient; ROC, the receiver operating characteristic.

(Figure 1). Thymomas are more likely to be enhanced on enhanced CT than thymic cysts, and we can see the ADC values are $0.82 \times 10^{-3} \mathrm{~mm}^{2} / \mathrm{s}$ and $3.27 \times 10^{-3} \mathrm{~mm}^{2} / \mathrm{s}$, respectively, on ADC map (Figure 2, Figure 3).

\section{Differential diagnostic performance of CT value and $\triangle C T$ value}

The CT values of plain CT, enhanced CT30s, enhanced CT120s, enhanced CT30s $\Delta$ CT, and enhanced CT120s $\Delta$ CT images of thymomas with diameters of $<5 \mathrm{~cm}$ were higher than those of thymic cysts, and those differences were statistically significant $(\mathrm{P}<0.05)$. The enhanced CT30sCT, enhanced CT120sCT, and enhanced CT120s $\Delta$ CT values of thymomas with diameters of $<3 \mathrm{~cm}$ were higher than those of thymic cysts, and those differences were statistically significant $(\mathrm{P}<0.05)$. The plain $\mathrm{CT}$ and enhanced $\mathrm{CT} 30 \mathrm{~s} \Delta \mathrm{CT}$ values of thymomas were also higher than those of thymic cysts, but the differences were not statistically significant $(\mathrm{P}>0.05)$. In patients with thymomas and thymic cysts with diameters of $<5$ and $<3 \mathrm{~cm}$, the AUC was highest for CT enhancement at 120s: the values were 0.94 and 0.95 , respectively (Figure 4).

\section{Diagnostic rates of CT, MRI, and CT combined with MRI for thymomas and thymic cysts}

In the differential diagnosis of thymomas and thymic cysts with diameters of $<5$ and $<3 \mathrm{~cm}$, the diagnostic rate of enhanced CT combined with MRI was higher than that of enhanced CT or MRI alone. In masses with diameters $<5 \mathrm{~cm}$, the diagnostic rates of enhanced CT, MRI, and enhanced CT combined with MRI for thymomas were $40.5 \%, 64.9 \%$, and $70.3 \%$, respectively, and those for thymic cysts were $37.5 \%, 62.5 \%$, and $71.9 \%$, respectively. In masses with diameters $<3 \mathrm{~cm}$, the diagnostic rates of enhanced CT, MRI, and enhanced CT combined with MRI for thymomas were $40.9 \%, 68.2 \%$ and $77.3 \%$, respectively, and those for thymic cysts were $32.0 \%, 64.0 \%$, and $68.0 \%$, respectively (Table 6).

\section{Discussion}

In clinical practice, anterior mediastinal thymic masses with diameters of $<5 \mathrm{~cm}$, especially those with diameters of $<3$ $\mathrm{cm}$, are difficult to identify before surgery. If patients with thymic cysts can be accurately diagnosed before surgery, unnecessary surgical intervention can be reduced. The fact that thymomas and thymic cysts have overlapping clinical features poses a challenge in terms of accurate preoperative diagnosis by clinicians. Percutaneous biopsy is recommended as the gold standard, but such biopsies are difficult to perform because these lesions are tiny and located behind the sternum or in front of the heart. Therefore, clinical diagnosis usually depends on imaging evaluation (15).

In our study, the proportions of women with thymomas and thymic cysts of diameters $<5$ and $<3 \mathrm{~cm}$ were higher than those of men, and the patients' median age ranged $55-57.5$ years, which is consistent with the results reported in previous literature (2). Thymic cysts are rarely associated with myasthenia gravis, while most patients who have thymomas also have myasthenia gravis. Whether their 

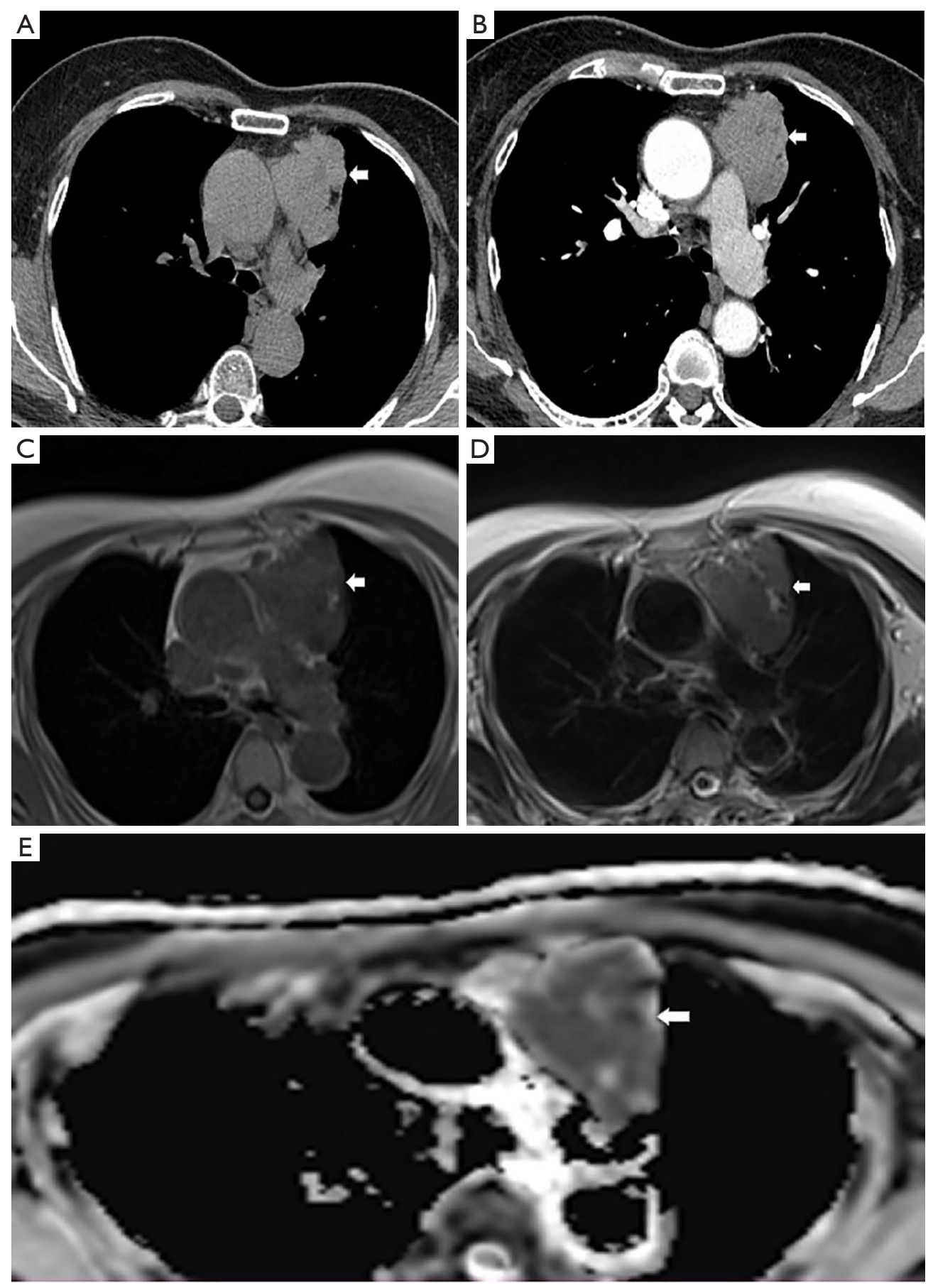

Figure 2 A 68-year-old woman with thymoma. (A) CT plain scan showed an iso-density (compared to chest wall muscle) mass on the left side of the anterior mediastinum, with uneven internal density. (B) Enhanced CT scan showed obvious enhancement of anterior mediastinal mass. (C) Axial T1WI showed an iso-signal (compared to chest wall muscle) mass in the anterior mediastinum. (D) Axial T2WI showed that the signal of anterior mediastinal mass was slightly uneven and slightly higher. (E) High signal was observed in the anterior mediastinum with ADC map. (the part indicated by the white arrows are thymomas). T1WI, T1-weighted imaging; T2WI, T2-weighted imaging; ADC, apparent diffusion coefficient. 

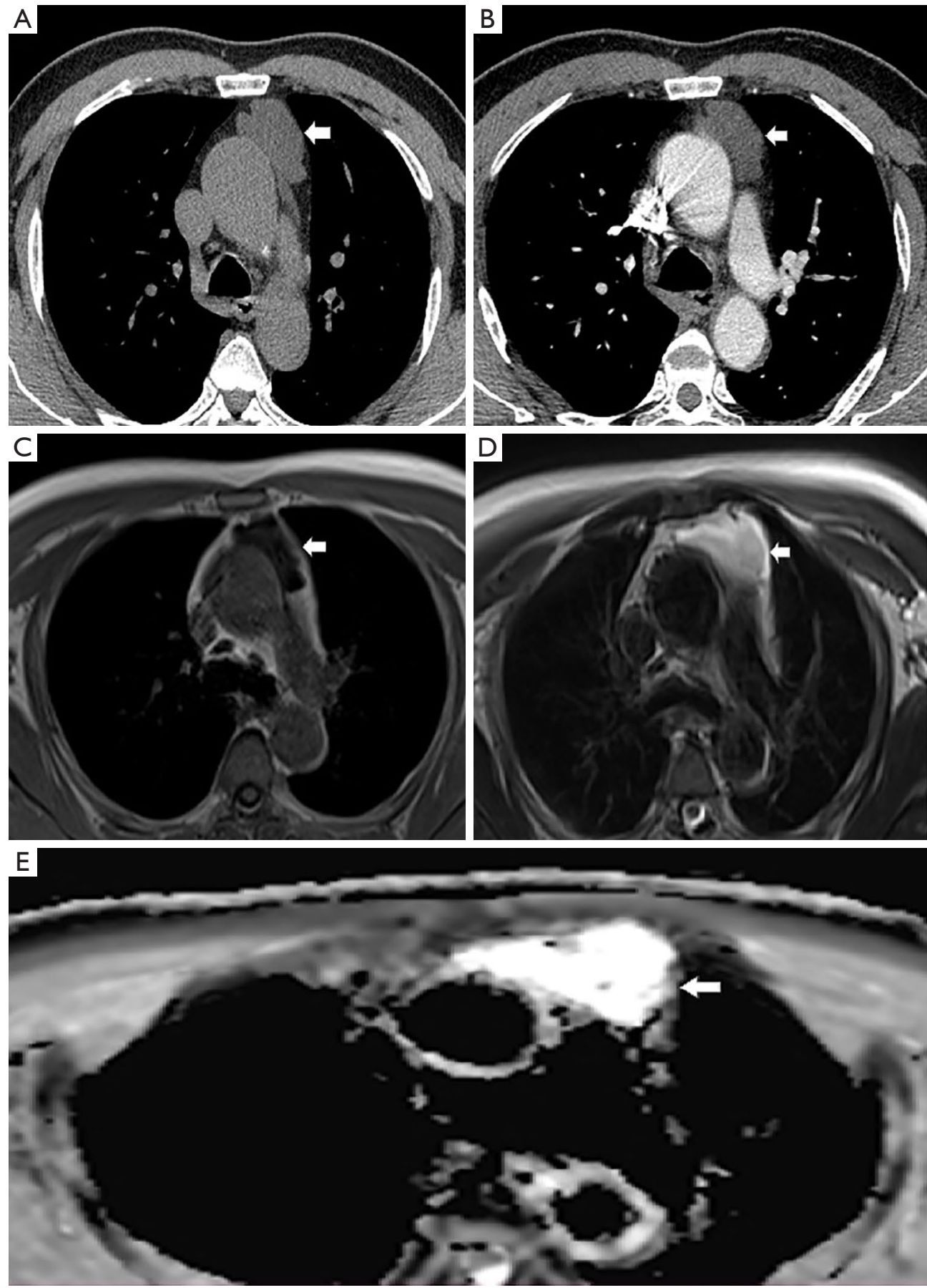

Figure 3 A 64-year-old man with thymic cyst. (A) CT plain scan showed a low-density (compared to chest wall muscle) mass on the left side of the anterior mediastinum, with uniform internal density. (B) CT enhanced scan showed no definite enhancement of the anterior mediastinal mass. (C) Axial T1WI showed a slightly low signal (compared to chest wall muscle) mass in the anterior mediastinum. (D) Axial T2WI showed that the anterior mediastinal mass was slightly uneven and obviously high signal. (E) No abnormal signal was observed in the anterior mediastinum with ADC map. (the part indicated by the white arrows are thymic cysts). T1WI, T1-weighted imaging; T2WI, T2weighted imaging; ADC, apparent diffusion coefficient. 
A

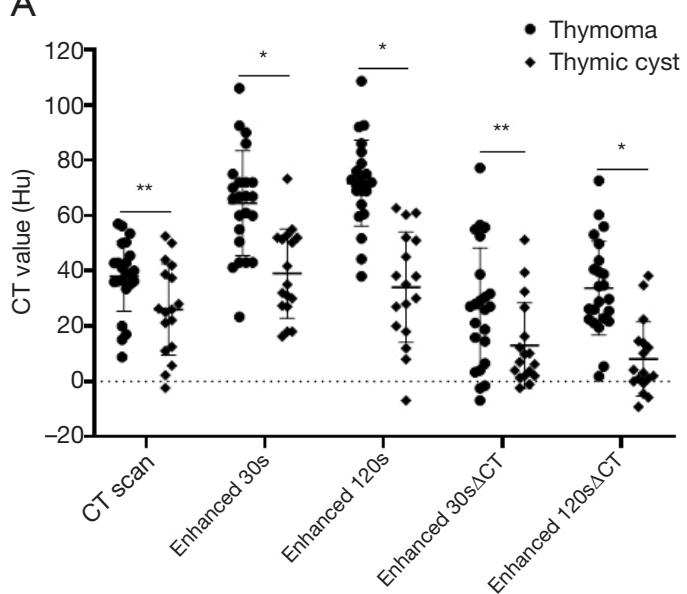

C

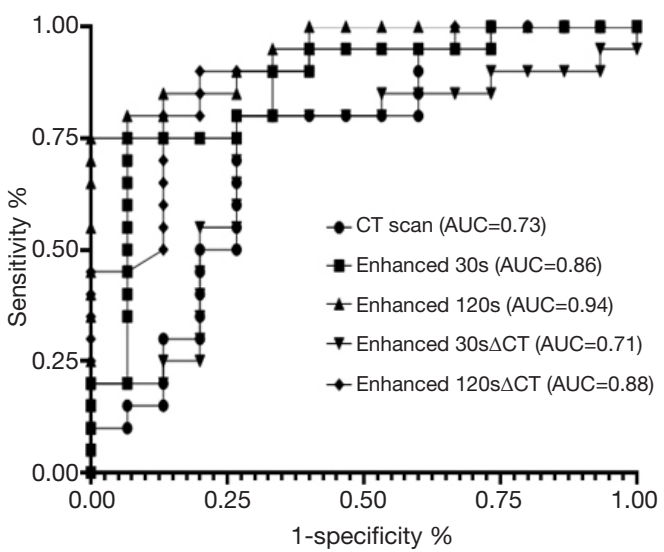

B

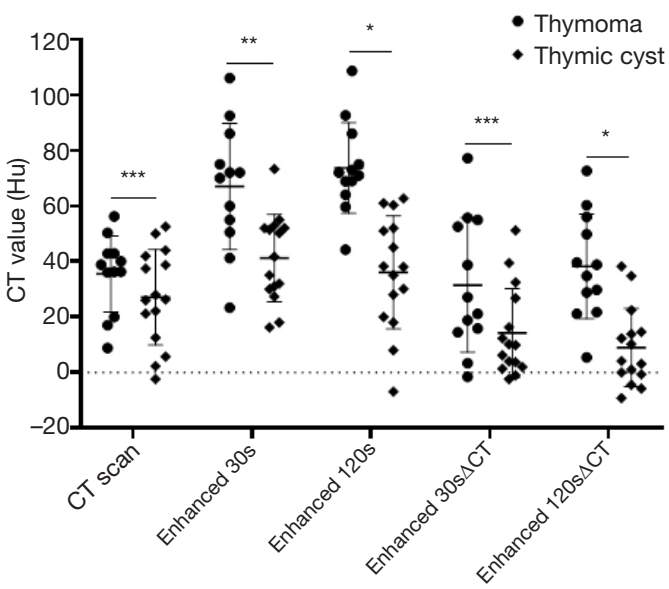

D

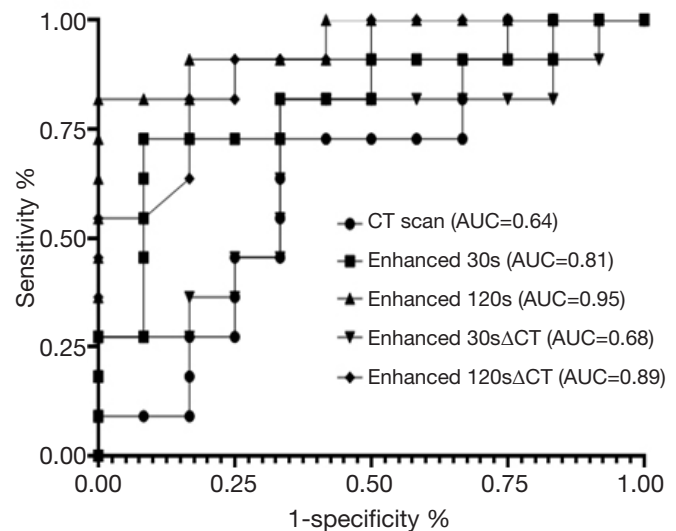

Figure 4 Scatter plots and ROC curves of the value of CT image types for differential diagnosis of thymomas and thymic cysts. Scatter plots of the value of plain CT, enhanced CT30s, enhanced CT120s, enhanced CT30s $\Delta$ CT, and enhanced CT120s $\Delta$ CT in diagnosis of thymomas and thymic cysts with diameters (A) $<5 \mathrm{~cm}$ and (B) $<3 \mathrm{~cm}$. ROC curves of the value of plain CT, enhanced CT30s, enhanced CT120s, enhanced CT30s $\Delta \mathrm{CT}$, and enhanced CT120s $\Delta \mathrm{CT}$ for the differential diagnosis of thymomas and thymic cysts with diameters $(\mathrm{C})<5 \mathrm{~cm}$ and (D) $<3 \mathrm{~cm}$. *, $\mathrm{P}<0.001$; **, $\mathrm{P}<0.05$; ***, $\mathrm{P}>0.05$. ROC, the receiver operating characteristic.

Table 6 Diagnostic rates of CT, MRI, and CT combined with MRI for thymic cysts and thymomas

\begin{tabular}{|c|c|c|c|c|c|}
\hline \multirow{2}{*}{ Diagnostic rate (\%) } & \multirow{2}{*}{ Imaging method } & \multicolumn{2}{|c|}{ Thymoma } & \multicolumn{2}{|c|}{ Thymic cyst } \\
\hline & & $<5 \mathrm{~cm}^{\dagger}$ & $<3 \mathrm{~cm}^{\ddagger}$ & $<5 \mathrm{~cm}^{\S}$ & $<3 \mathrm{~cm}^{\pi}$ \\
\hline \multirow{3}{*}{ Positive rate } & CT & $15(40.5)$ & $9(40.9)$ & $12(37.5)$ & $8(32.0)$ \\
\hline & MRI & $24(64.9)$ & $15(68.2)$ & $20(62.5)$ & $16(64.0)$ \\
\hline & CT or MRI & $26(70.3)$ & $17(77.3)$ & $23(71.9)$ & $17(68.0)$ \\
\hline \multirow[t]{2}{*}{ Negative rate } & MRI & $13(35.1)$ & 7 (31.8) & $12(37.5)$ & $9(36.0)$ \\
\hline & $\mathrm{CT}$ or MRI & $11(29.7)$ & $5(22.7)$ & $9(28.1)$ & $8(32.0)$ \\
\hline
\end{tabular}

${ }^{\dagger}, \mathrm{n}=37 ;{ }^{\ddagger}, \mathrm{n}=22 ;{ }^{\S}, \mathrm{n}=32 ;{ }^{\natural}, \mathrm{n}=25 . \mathrm{CT}$, computed tomography; MRI, magnetic resonance imaging. 
diameter is $<5$ or $<3 \mathrm{~cm}$, most thymomas have a rough outline, while most thymic cysts have a smooth outline. The largest proportions of lesions of both types had clearbounded and irregular shapes, matching the results of previous studies $(1,7,16,17)$. On MRI, we compared the signal strength of the lesion with that of the muscle and divided the mass into low-, iso-, and high-signal groups. In our patients, thymic cysts with diameters of $<5$ and $<3 \mathrm{~cm}$ appear as iso-signal or high-signal on T1WI and T2WI. However, thymomas mostly show iso-signal intensity on T1WI and T2WI. Previous studies have revealed that thymic cysts show high signal intensity on T2WI, sometimes because of hemorrhage infection or high protein levels. High- and iso-signal intensity on T1WI have been shown consistent with our research results. In our study $(18,19)$, among thymomas and thymic cysts with diameters of $<5 \mathrm{~cm}$, thymomas and thymic cysts had the largest proportions of iso- or high-signal and lowsignal intensity on DWI, respectively, and the difference was statistically significant. However, in patients with thymomas and thymic cysts with diameters $<3 \mathrm{~cm}$, the two lesion types both had the largest proportion of iso-signal intensity on DWI, and the difference was not statistically significant. We made the ROC curves out of ADC value and found that when the lesion is less than $5 \mathrm{~cm}$ and $3 \mathrm{~cm}$, ADC $>2.68 \times 10^{-3} \mathrm{~mm}^{2} / \mathrm{s}$ and $>2.55 \times 10^{-3} \mathrm{~mm}^{2} / \mathrm{s}$ are more prone to thymic cysts. It is reported in other literature that ADC values $>2.5 \times 10^{-3} \mathrm{~mm}^{2} / \mathrm{s}$ tend to be thymic cysts (9), which is corresponding to our results.

Dynamic enhancement CT can reflect a lesion's hemodynamic characteristics. The pathophysiological characteristics of thymomas and thymic cysts are completely different. In our study, in patients with mass diameters of $<3 \mathrm{~cm}$, the enhanced CT30sCT, enhanced CT120sCT, and enhanced CT120s $\Delta$ CT values differed significantly between thymomas and thymic cysts. Thymic cysts showed only marginal enhancement, and thymomas showed more uneven enhancement, and the enhanced CT values of thymomas were significantly higher than those of thymic cysts. We reached a consistent conclusion in a previous study (20). In patients with mass diameters of $<5 \mathrm{~cm}$, the plain $\mathrm{CT}$, enhanced CT30sCT, enhanced CT120sCT, enhanced CT30s $\Delta$ CT, and enhanced CT120s $\Delta$ CT values differed significantly between thymomas and thymic cysts. This indicates that it is difficult to distinguish between thymomas and thymic cysts with diameters of $<3 \mathrm{~cm}$ on plain CT, which is consistent with the results of previous studies $(4,20)$. On the ROC curves characterized by CT values, the AUCs of thymomas and thymic cysts with diameters of $<5 \mathrm{~cm}$ and $<3 \mathrm{~cm}$ had the largest values (0.94 and 0.95 , respectively) on 120s enhancement CT. This indicates that thymomas can be accurately distinguished from thymic cysts on 120 s enhancement CT.

In our study, the diagnostic rates of CT combined with MRI for thymomas and thymic cysts with diameters of $<5 \mathrm{~cm}$ were $70.3 \%$ and $71.9 \%$, respectively, and the diagnostic rates for thymomas and thymic cysts with diameters $<3 \mathrm{~cm}$ were $77.3 \%$ and $68.0 \%$, respectively. Although the diagnosis rate was not very high, different doctors or even the same doctor may have variable diagnostic effects on the same disease. Therefore, most of these diagnoses are not deterministic, but the imaging diagnosis indicates that the lesion is more likely to be considered as a certain type of mass. Moreover, our research shows that the diagnostic rate of CT combined with MRI is higher than that of enhanced CT or MRI alone for thymomas and thymic cysts. Therefore, MRI can be useful for diagnosing thymic diseases, and it may provide a supplement to $\mathrm{CT}$ in daily imaging diagnosis.

Our study is limited in that it is a retrospective study and is thus susceptible to potential selection bias. However, in our study, we used enhanced CT combined with MRI for the preoperative differential diagnosis of thymomas and thymic cysts with diameters of $<5 \mathrm{~cm}$, performed a subgroup analysis, and then explored the diagnostic efficiency of thymomas and thymic cysts by CT combined with MRI.

In summary, the accuracy of enhanced CT or MRI alone in the differential diagnosis of thymomas and thymic cysts is limited, whereas the diagnostic efficiency of enhanced CT combined with MRI is improved for both mass types. Therefore, in the preoperative diagnosis of anterior mediastinal masses, enhanced CT combined with MRI examination can improve the accuracy of preoperative diagnosis, and unnecessary surgery and injury can be avoided.

\section{Acknowledgments}

Thanks Richard Lipkin, the English editor in Certificate of Editing, for the language editing of our article.

Funding: This work was supported by the National Natural Science Foundation of China Youth Program (81801603).

\section{Footnote}

Reporting Checklist: The authors have completed the 
STARD reporting checklist. Available at https://dx.doi. org/10.21037/tcr-21-96

Data Sharing Statement: available at https://dx.doi. org/10.21037/tcr-21-96

Conflicts of Interest: All authors have completed the ICMJE uniform disclosure form (available at https://dx.doi. org/10.21037/tcr-21-96). All authors report National Natural Science Foundation of China Youth Program (81801603) Funding for this study.

Ethical Statement: The authors are accountable for all aspects of the work in ensuring that questions related to the accuracy or integrity of any part of the work are appropriately investigated and resolved. The study was conducted in accordance with the Declaration of Helsinki (as revised in 2013). The study does not require ethical approval. Because it is a retrospective study, it only needs to review the patient's preoperative imaging data and postoperative pathological results, and does not need to use the patient's blood and tissue specimens, so it does not involve ethical issues and individual consent for this retrospective analysis was waived.

Open Access Statement: This is an Open Access article distributed in accordance with the Creative Commons Attribution-NonCommercial-NoDerivs 4.0 International License (CC BY-NC-ND 4.0), which permits the noncommercial replication and distribution of the article with the strict proviso that no changes or edits are made and the original work is properly cited (including links to both the formal publication through the relevant DOI and the license). See: https://creativecommons.org/licenses/by-nc-nd/4.0/.

\section{References}

1. Hayes SA, Huang J, Plodkowski AJ, et al. Preoperative computed tomography findings predict surgical resectability of thymoma. J Thorac Oncol 2014;9:1023-30.

2. Araki T, Sholl LM, Gerbaudo VH, et al. Intrathymic cyst: Clinical and radiological features in surgically resected cases. Clin Radiol 2014;69:732-8.

3. Scagliori E, Evangelista L, Panunzio A, et al. Conflicting or complementary role of computed tomography (CT) and positron emission tomography (PET)/CT in the assessment of thymic cancer and thymoma: our experience and literature review. Thorac Cancer 2015;6:433-42.
4. Yoon $\mathrm{SH}$, Choi $\mathrm{SH}$, Kang $\mathrm{CH}$, et al. Incidental Anterior Mediastinal Nodular Lesions on Chest CT in Asymptomatic Subjects. J Thorac Oncol 2018;13:359-66.

5. Kent MS, Wang T, Gangadharan SP, et al. What is the prevalence of a "nontherapeutic" thymectomy? Ann Thorac Surg 2014;97:276-82; discussion 282.

6. Gochi F, Omasa M, Yamada T, et al. Factors affecting the preoperative diagnosis of anterior mediastinal cysts. Gen Thorac Cardiovasc Surg 2015;63:349-53.

7. Jung W, Cho S, Yum S, et al. Differentiating thymoma from thymic cyst in anterior mediastinal abnormalities smaller than $3 \mathrm{~cm}$. J Thorac Dis 2020;12:1357-65.

8. Hwang EJ, Paek M, Yoon SH, et al. Quantitative Thoracic Magnetic Resonance Criteria for the Differentiation of Cysts from Solid Masses in the Anterior Mediastinum. Korean J Radiol 2019;20:854-61.

9. Shin KE, Yi CA, Kim TS, et al. Diffusion-weighted MRI for distinguishing non-neoplastic cysts from solid masses in the mediastinum: problem-solving in mediastinal masses of indeterminate internal characteristics on CT. Eur Radiol 2014;24:677-84.

10. Padhani AR, Liu G, Koh DM, et al. Diffusionweighted magnetic resonance imaging as a cancer biomarker: consensus and recommendations. Neoplasia 2009;11:102-25.

11. Priola AM, Gned D, Veltri A, et al. Chemical shift and diffusion-weighted magnetic resonance imaging of the anterior mediastinum in oncology: Current clinical applications in qualitative and quantitative assessment. Crit Rev Oncol Hematol 2016;98:335-57.

12. Yi CA, Jeon TY, Lee KS, et al. 3-T MRI: usefulness for evaluating primary lung cancer and small nodules in lobes not containing primary tumors. AJR Am J Roentgenol 2007;189:386-92.

13. Li HR, Gao J, Jin C, et al. Comparison between CT and MRI in the Diagnostic Accuracy of Thymic Masses. J Cancer 2019;10:3208-13.

14. Matteo Zanichelli, Manuela Gozzi, Mario Bertolani. Radiologic Diagnosis: X-ray, CT and MRI. In: Corrado Lavini, Cesar A.Moran, Uliano Morandi, Rudolf Schoenhuber, editors. Thymus Gland Pathology. Italy: Springer-Verlag; 2008. p .99-105.

15. Li X, Han X, Sun W, et al. Preoperative misdiagnosis analysis and accurate distinguish intrathymic cyst from small thymoma on computed tomography. J Thorac Dis 2016;8:2086-92.

16. Nam JG, Goo JM, Park CM, et al. Age- and genderspecific disease distribution and the diagnostic accuracy 
of CT for resected anterior mediastinal lesions. Thorac Cancer 2019;10:1378-87.

17. Tomiyama N, Honda O, Tsubamoto M, et al. Anterior mediastinal tumors: diagnostic accuracy of CT and MRI. Eur J Radiol 2009;69:280-8.

18. Park JW, Jeong WG, Lee JE, et al. Pictorial Review of Mediastinal Masses with an Emphasis on Magnetic Resonance Imaging. Korean J Radiol 2021;22:139-54.

Cite this article as: Zhang X, Zhang R, Cao Y, Wang X, Chen $\mathrm{Y}$. The value of enhanced computed tomography combined with magnetic resonance imaging in the differential diagnosis of thymomas and thymic cysts before operation. Transl Cancer Res 2021;10(6):2777-2789. doi: 10.21037/tcr-21-96
19. Jeung MY, Gasser B, Gangi A, et al. Imaging of cystic masses of the mediastinum. Radiographics 2002;22 Spec No:S79-93.

20. Zhonggao J, YiJiao W, Yongfeng $W$, et al. Multislice computed tomography performance in differential diagnosis of high-density thymic cyst and thymoma in lesions less than $3 \mathrm{~cm}$. Thorac Cancer 2018;9:1300-4. 